\title{
Os impactos na autoestima de mulheres mastectomizadas: Revisão integrativa
}

\author{
Impacts on the self-esteem of mastectomized women: An integrative review \\ Impactos en la autoestima de mujeres mastectomizadas: Una revisión integradora
}

Recebido: 18/05/2021 | Revisado: 28/05/2021 | Aceito: 29/05/2021 | Publicado: 12/06/2021

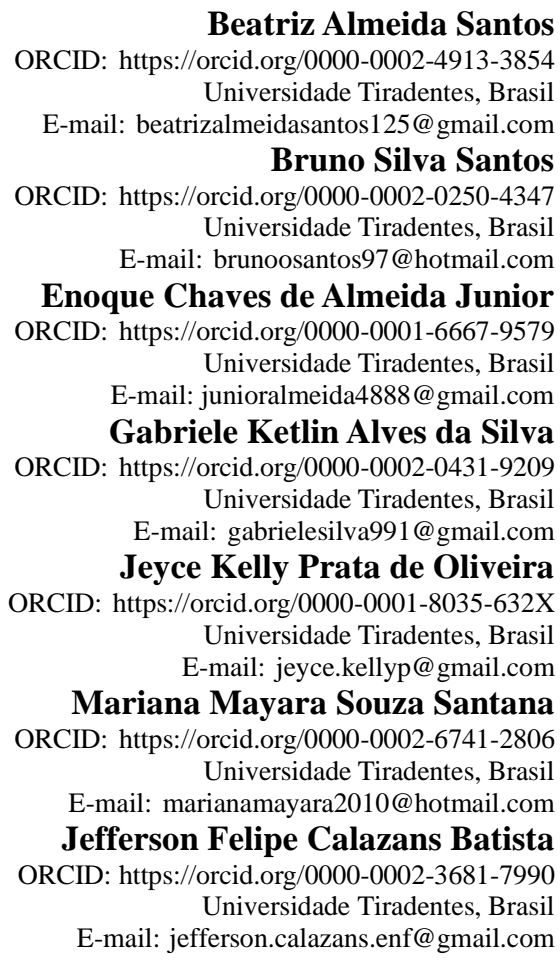

\section{Resumo}

Mulheres acometidas pelo câncer de mama são submetidas constantemente a mastectomia, como principal forma de tratamento. Nesse momento, diversos sentimentos perpassam desde o diagnóstico ao tratamento, em razão da importância desse órgão à figura feminina. Para tanto, buscou-se analisar os aspectos envolvidos na autoestima de mulheres com câncer de mama e/ou submetidas à mastectomia. Para o alcance do objetivo, foi realizado um estudo de revisão integrativa da literatura de natureza qualitativa e caráter descritivo. Após leitura integral de 60 estudos indexados nas bases de dados da SciELO, BVS, LILACS, PubMed e Science Direct, obtidos a partir do cruzamento entre os descritores, selecionou-se 14 artigos científicos nos idiomas inglês (50\%), português (35,7\%) e espanhol (14,3\%), com predominância no ano de 2019 (35,8\%), seguido de $2018(28,6 \%)$ e 2017 (21,4\%), enquanto 2020 e 2021 apresentaram o mesmo quantitativo $(7,1 \%)$. Nesse contexto, dentre os sentimentos negativos mais prevalentes em mulheres mastectomizadas, destacam-se sensações de inferioridade, sintomas de depressão, vergonha, isolamento, pessimismo, alterações na imagem corporal e satisfação sexual. Portanto, ressalta-se a importância da rede de apoio e da reconstrução mamária, por refletir positivamente na diminuição dos sintomas desencadeados nas diversas situações em que as mulheres perpassam durante sua luta contra o câncer de mama.

Palavras-chave: Autoestima; Câncer de mama; Mastectomia; Mulher.

\begin{abstract}
Women affected by breast cancer are constantly subjected to mastectomy, as the main form of treatment. At that moment, several feelings run from diagnosis to treatment, due to the importance of this organ to the female figure. To this end, we sought to analyze the aspects involved in the self-esteem of women with breast cancer and/or undergoing mastectomy. To achieve the objective, a study of integrative literature review of qualitative nature and descriptive character was carried out. After full reading of 60 studies indexed in the SciELO, BVS, LILACS, PubMed and Science Direct databases, obtained from the crossing between descriptors, 14 scientific articles were selected in English (50\%), Portuguese (35, 7\%) and Spanish (14.3\%), with a predominance in 2019 (35.8\%), followed by 2018 (28.6\%) and 2017 (21.4\%), while 2020 and 2021 presented the same quantitative (7.1\%). In this context, among the most prevalent negative feelings in mastectomized women, feelings of inferiority, symptoms of depression, shame,
\end{abstract}


isolation, pessimism, changes in body image and sexual satisfaction stand out. Therefore, the importance of the support network and breast reconstruction is emphasized, as it reflects positively on the reduction of symptoms triggered in the different situations in which women experience during their fight against breast cancer.

Keywords: Self esteem; Breast cancer; Mastectomy; Woman.

\section{Resumen}

Las mujeres afectadas por el cáncer de mama son sometidas constantemente a mastectomía, como principal forma de tratamiento. En ese momento, varios sentimientos corren desde el diagnóstico hasta el tratamiento, debido a la importancia de este órgano para la figura femenina. Para ello, se buscó analizar los aspectos involucrados en la autoestima de mujeres con cáncer de mama y/o sometidas a mastectomía. Para lograr el objetivo, se realizó un estudio de revisión integradora de la literatura de carácter cualitativo y de carácter descriptivo. Luego de la lectura completa de 60 estudios indexados en las bases de datos SciELO, BVS, LILACS, PubMed y Science Direct, obtenidos del cruce entre descriptores, se seleccionaron 14 artículos científicos en inglés (50\%), portugués (35,7\%) y español (14,3\%), con predominio en 2019 (35,8\%), seguido de $2018(28,6 \%)$ y $2017(21,4 \%)$, mientras que 2020 y 2021 presentaron el mismo cuantitativo $(7,1 \%)$. En este contexto, entre los sentimientos negativos más prevalentes en las mujeres mastectomizadas destacan los sentimientos de inferioridad, síntomas de depresión, vergüenza, aislamiento, pesimismo, cambios en la imagen corporal y satisfacción sexual. Por ello, se enfatiza la importancia de la red de apoyo y reconstrucción mamaria, ya que se refleja positivamente en la reducción de los síntomas desencadenados en las diferentes situaciones que viven las mujeres durante su lucha contra el cáncer de mama.

Palabras clave: Autoestima; Cáncer de mama; Mastectomía; Mujer.

\section{Introdução}

Câncer de Mama (CA), bem como as demais neoplasias malignas, são doenças de grande incidência mundial, resultadas da multiplicação de células anormais. Geralmente, essa neoplasia ocorre nos ductos e glóbulos mamários, seja por fatores genéticos, fisiológicos ou por exposição aos fatores ambientais (Araújo et al., 2020; Prates et al., 2017). Diante desse cenário, o CA encontra-se no topo dos cânceres mais recorrentes e temidos entre as mulheres, resultando em alteração da imagem corporal, impactos na autoestima e na qualidade de vida (Oliveira et al., 2019).

De acordo com o Instituto Nacional de Câncer, o câncer de mama é o segundo tipo mais incidente nas mulheres em todo o mundo, responsável por $25 \%$ do total de novos casos da doença a cada ano. Atualmente, no Brasil, há incidência de 596 mil casos de câncer, sendo as mulheres 300.870, com o CA de mama correspondendo a 57.960 (Junqueira \& Santos, 2020). Além disso, estima-se 66.280 casos de CA de mama feminina para cada ano do triênio 2020-2022, tornando-se, dessa forma, um importante índice para as políticas públicas de saúde de todo o país (Nascimento et al., 2015; Brasil, 2020).

Segundo Rocha et al. (2018), quando o diagnóstico dessa doença é confirmado, emoções e sentimentos negativos ao longo do processo de adoecimento e tratamento surgem, causando uma baixa valorização tanto no aspecto emocional quanto no anatômico na mulher. Embora exista o sentimento de medo, se faz necessário iniciar o tratamento na fase inicial da doença, visando obter melhores resultados por meio das diversas modalidades terapêuticas ofertadas pelos serviços de saúde, de modo que seja menos agressivo ao seu organismo (Gomes, Lima \& Santos, 2021).

Nesse contexto, o tratamento pode ser realizado clínico ou cirurgicamente. Na abordagem clínica, tem-se a radioterapia, hormonioterapia e quimioterapia, enquanto na cirúrgica, por sua vez, baseia-se na tumorectomia, que consiste na retirada do tumor, assim como, pela quadrantectomia e mastectomia, definidos, respectivamente, pela remoção parcial e total da mama (Prates et al., 2014).

A mastectomia é uma das abordagens terapêuticas mais executadas entre as mulheres com o tumor mamário, podendo ser simples, quando ocorre somente a retirada das glândulas mamárias e da aponeurose no músculo peitoral maior, bem como radical, na qual a glândula mamária total do músculo peitoral e dos linfonodos axilares é removida completamente (Lima et al., 2018). Somado a isso, Godoy et al. (2016), enfatizam que, em muitos casos, esse procedimento cirúrgico é realizado como medida preventiva, principalmente, quando existe um risco elevado para o desenvolvimento da doença. 
A notificação da cirurgia para remoção do tumor apresenta um grande impacto para à saúde mental da mulher, tendo em vista que a mama é um órgão sexual e que tem por representação a sexualidade e, ao mesmo tempo, integra a imagem da constituição da feminilidade (Noal \& Bergamaschi, 2017). Diante disso, Salimena et al. (2012), abordam que esta perspectiva gera uma grande ameaça para a autovalorização e bem estar socioemocional das mulheres, devido aos efeitos que essa neoplasia causa na sua autoimagem.

Em concordância, Cammarota et al. (2019), discutem que a dor, alteração na sensação tátil do seio, desconfortos na área das mamas e comprometimento da funcionalidade do membro superior após a dissecção dos linfonodos axilares são os principais sintomas que podem surgir após o procedimento cirúrgico. Além disso, a perda de uma ou ambas as mamas, aumenta os riscos para depressão, baixa autoestima, impotência, inferioridade, perda da sua atratividade física e medo da recidiva (Lima et al., 2018). Dessa forma, avaliar o efeito do tipo de tratamento do câncer de mama na imagem corporal e na autoestima é de extrema relevância, uma vez que as mulheres participam da tomada de decisão quanto ao tratamento (Prates et al., 2019). Os sentimentos após a mastectomia surgem inesperadamente, sejam eles positivos ou negativos.

Devido às alterações ocasionadas durante o tratamento e por se tratar de uma vivência dolorosa, a qual resulta em consequências na autoestima, torna-se imprescindível uma rede de apoio constituída por indivíduos que possui algum vínculo com a paciente, tendo em vista sua contribuição para a redução dos impactos ocasionados pelo CA. Nesta perspectiva, destacase também a equipe de saúde, que identificam como pontos primordiais para o atendimento, a humanização e empatia, através do contato, comunicação, instruções e do cuidado holístico, de modo a proporcionar uma relação de apoio e confiabilidade com as mulheres mastectomizadas (Santos et al., 2017; Ribeiro \& Marteleto, 2018).

O câncer de mama é um dos males mais comuns entre as mulheres e que pode resultar em grandes impactos no âmbito físico, psicológico e social, principalmente quando associado ao processo de retirada da mama. Com base na importância do tratamento por mastectomia e reconstrução mamária após cirurgia para a qualidade de vida e melhor aceitação da imagem corporal das mulheres, tem-se como justificativa a elaboração do presente estudo como meio de levantar evidências científicas acerca da eficácia desse tratamento, assim como, dos sentimentos que perpassam durante o enfrentamento do CA. Dessa forma, esta pesquisa tem o objetivo de analisar os aspectos envolvidos na autoestima de mulheres com câncer de mama e/ou submetidas à mastectomia.

\section{Metodologia}

Trata-se de uma revisão integrativa da literatura de natureza qualitativa e caráter descritivo. Segundo Pereira et al. (2018), os aspectos metodológicos do estudo qualitativo permitem ao pesquisador, emitir suas próprias concepções referente ao fenômeno de estudo interpretado. Para o estudo, seguiram-se as seguintes etapas da revisão integrativa: elaboração da pergunta norteadora, busca na literatura, coleta de dados, análise dos artigos, discussão dos resultados e apresentação da revisão (Souza, Silva \& Carvalho, 2010).

O levantamento bibliográfico foi executado no mês de março de 2021 mediante busca no banco de dados da Scientific Electronic Library Online (SciELO), Biblioteca Virtual em Saúde (BVS), Literatura Latino-Americana e do Caribe em Ciências da Saúde (LILACS), National Library of Medicine (PubMed) e Science Direct. A ferramenta de busca Google Acadêmico foi utilizada de forma isolada para refinamento das buscas.

As estratégias de busca foram realizadas a partir do cruzamento dos Descritores em Ciências da Saúde (DeCS/MeSH): “Autoestima", "Câncer de mama", "Mastectomia" e "Mulher" e suas respectivas versões em inglês (Quadro 1) com o operador booleano "AND". 
Os critérios de inclusão para seleção dos estudos foram: artigos publicados na íntegra entre os anos de 2016 a 2021 , nos idiomas português, inglês e espanhol, e estudos no formato original. Para tanto, excluíram-se teses, monografias, dissertações, publicações em anais de eventos e artigos em duplicata.

Quadro 1 - Estratégias de busca para as bases SciELO, BVS, LILACS, PubMed e Science Direct.

\begin{tabular}{|c|c|}
\hline Estratégias de busca & \multirow{2}{*}{$\mathbf{N}$} \\
\hline Português & \\
\hline "mastectomia" AND “autoestima" AND "câncer de mama" & 19 \\
\hline \multicolumn{2}{|l|}{ Inglês } \\
\hline "mastectomy" AND “self-esteem” AND “woman" & 1421 \\
\hline "mastectomy" AND "self-esteem" AND "breast cancer" & 1805 \\
\hline
\end{tabular}

Fonte: Autores (2021).

Os artigos foram selecionados em primeiro plano por leitura do título, seguido do resumo e, por fim, do texto completo (Figura 1). A seleção dos artigos foi realizada por dois revisores, destinado um terceiro pesquisador como juiz, para decisão final nos casos de discrepância decisória.

Figura 1 - Representação esquemática das etapas de seleção dos artigos, 2021.

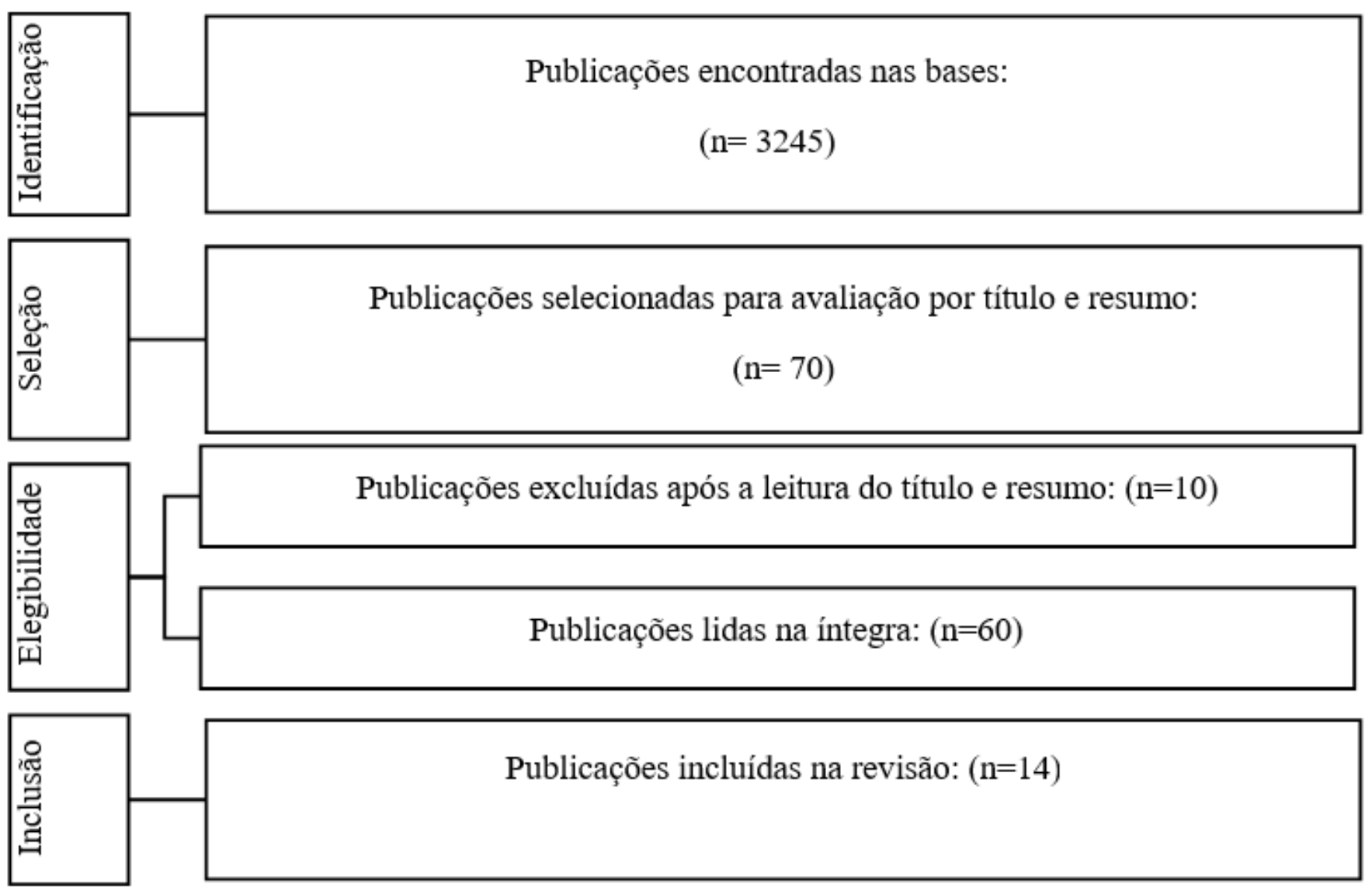


Os títulos dos estudos incluídos na revisão foram organizados em uma planilha do software Microsoft Excel by Office 2019, através de um instrumento de coleta de dados, destacando-se: ano de publicação, autores, localização geográfica, qualis da revista, Fator de Impacto (FI), objetivo e principais resultados.

Após análise completa dos artigos e confecção da planilha, as informações como: autor, ano de publicação, base de dados, periódico, Fator de Impacto (FI), objetivo e resumo dos principais resultados serão dispostos em um quadro síntese (Quadro 2).

\section{Resultados e Discussão}

A partir da triagem inicial executada nos bancos de dados, incluíram-se nesta revisão 14 estudos e desse total, cinco foram realizados no Brasil, quatro na Polônia e dois na Espanha, enquanto China, Turquia e Marrocos, obtiveram apenas um estudo em cada país.

A distribuição dos estudos demonstrou predominância na base do PubMed com sete artigos, enquanto na SciELO, Science Direct e BVS, indexavam-se quatro, dois e uma publicação, respectivamente.

Foram identificadas publicações, nos idiomas inglês (50\%), português $(35,7 \%)$ e espanhol $(14,3 \%)$. Em relação ao ano de publicação, houve predominância do ano de 2019 (35,8\%), seguido de 2018 (28,6\%) e 2017 (21,4\%), enquanto 2020 e 2021 apresentaram o mesmo quantitativo $(7,1 \%)$.

Quadro 2 - Esquematização dos estudos incluídos no estudo.

\begin{tabular}{|c|c|c|c|c|c|}
\hline $\begin{array}{l}\text { Autores e } \\
\text { ano }\end{array}$ & $\begin{array}{c}\text { Base de } \\
\text { dados }\end{array}$ & $\begin{array}{c}\text { Local do } \\
\text { estudo }\end{array}$ & FI & Objetivo & Principais resultados \\
\hline $\begin{array}{l}\text { OLIVEIRA } \\
\text { et al., } 2019\end{array}$ & BVS & Brasil & $\begin{array}{l}\text { Não } \\
\text { disponível }\end{array}$ & $\begin{array}{l}\text { Compreender } r a \\
\text { percepção da imagem } \\
\text { corporal de mulheres } \\
\text { mastectomizadas, com ou } \\
\text { sem reconstrução } \\
\text { mamária. }\end{array}$ & $\begin{array}{l}\text { O cuidado com as mulheres acometidas pelo CA de } \\
\text { mama demanda uma assistência multiprofissional, } \\
\text { para auxiliar o processo de enfrentamento do câncer e } \\
\text { na reabilitação para além das limitações físicas, } \\
\text { visando uma melhor adaptação ao "novo corpo", } \\
\text { refletindo na melhoria da qualidade de vida. }\end{array}$ \\
\hline $\begin{array}{l}\text { ROCHA et } \\
\text { al., } 2019\end{array}$ & SciELO & Brasil & 0,669 & $\begin{array}{l}\text { Descrever os sentimentos } \\
\text { que emergem das } \\
\text { mulheres com câncer de } \\
\text { mama, submetidas à } \\
\text { mastectomia total. }\end{array}$ & $\begin{array}{l}\text { A amostra foi composta por } 12 \text { mulheres que se } \\
\text { declararam de cor parda, casada, com baixo grau de } \\
\text { escolaridade, de religião católica e dona-de-casa. Os } \\
\text { resultados mostraram que a participação da família, } \\
\text { amigos e crença religiosa, contribuem para os } \\
\text { sentimentos positivos a essas mulheres. }\end{array}$ \\
\hline $\begin{array}{l}\text { BOING et } \\
\text { al., } 2019\end{array}$ & SciELO & Brasil & 1,748 & $\begin{array}{l}\text { Analisar os fatores } \\
\text { associados à presença de } \\
\text { sintomas depressivos em } \\
\text { mulheres após o câncer } \\
\text { de mama. }\end{array}$ & $\begin{array}{l}\text { A associação entre os sintomas depressivos e o grupo } \\
\text { de mulheres entre } 40 \text { e } 60 \text { anos, e, aquelas que tinham } \\
\text { outra doença, que realizaram a mastectomia e que } \\
\text { sofriam de linfedema, apresentaram redução média } \\
\text { na autoestima. As que apresentavam baixo nível de } \\
\text { escolaridade relataram mais sintomas depressivos. }\end{array}$ \\
\hline $\begin{array}{l}\text { SÁNCHEZ } \\
\text { et al., } 2021\end{array}$ & PubMed & Espanha & 2.849 & $\begin{array}{l}\text { Identificar e examinar as } \\
\text { intervenções } \\
\text { implementadas com o } \\
\text { objetivo de potencializar } \\
\text { ambas as variáveis nesta } \\
\text { população. }\end{array}$ & $\begin{array}{l}\text { Agruparam-se três intervenções, sendo elas: Terapias } \\
\text { de grupo, Terapias de atividade física e Tratamentos } \\
\text { cosméticos e de beleza. Tais intervenções mostraram- } \\
\text { se efetivas na melhoria da autoestima das mulheres, } \\
\text { autopercepção da feminilidade e autoconfiança. }\end{array}$ \\
\hline
\end{tabular}




\begin{tabular}{|c|c|c|c|c|c|}
\hline $\begin{array}{l}\text { JABŁOŃSK } \\
\text { I et al., } 2018\end{array}$ & PubMed & Polônia & 1.097 & $\begin{array}{l}\text { Verificar hipóteses } \\
\text { relacionadas ao câncer de } \\
\text { mama, realização da } \\
\text { mastectomia e avaliar as } \\
\text { variáveis demográficas: } \\
\text { IMC, tamanho das } \\
\text { mamas e avaliação da } \\
\text { cicatriz após cirurgia. }\end{array}$ & $\begin{array}{l}\text { Mulheres mastectomizadas, mostraram resultados } \\
\text { significativamente mais baixos nas duas categorias } \\
\text { do questionário J-C: Aceitação corporal e vivência da } \\
\text { intimidade. Substancialmente valor médio mais alto } \\
\text { foi registrado para a categoria que representa a } \\
\text { manifestação da feminilidade em favor das pacientes } \\
\text { tratadas quando comparadas ao grupo controle de } \\
\text { mulheres saudáveis. }\end{array}$ \\
\hline $\begin{array}{l}\text { MAHARJ et } \\
\text { al., } 2018\end{array}$ & PubMed & China & 2.52 & $\begin{array}{l}\text { Determinar a qualidade } \\
\text { de vida de mulheres } \\
\text { nepalesas após a } \\
\text { mastectomia. }\end{array}$ & $\begin{array}{l}\text { No Módulo Específico para mamas, } 100 \% \text { das } \\
\text { entrevistadas tiveram um ruim desempenho em } \\
\text { relação à função e prazer sexual. Enquanto no Breast } \\
\text { Specific Module, percebeu-se que os efeitos colaterais } \\
\text { da terapia sistêmica mostraram fortes estatísticas nas } \\
\text { associações entre idade, ocupação, educação, estado } \\
\text { civil, uso de prótese mamária e comorbidades. }\end{array}$ \\
\hline $\begin{array}{l}\text { AYGIN; } \\
\text { CENGIZ, H, } \\
2018\end{array}$ & PubMed & Turquia & 4.988 & 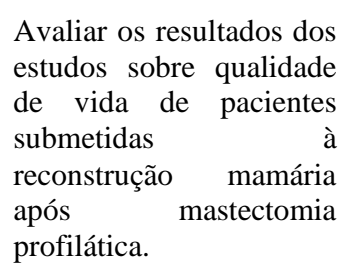 & $\begin{array}{l}\text { Embora tenham relatado quadros de dor e problemas } \\
\text { na sexualidade após mastectomia, os estudos } \\
\text { evidenciaram que grande parte das pacientes ficou } \\
\text { satisfeita com o resultado da cirurgia. }\end{array}$ \\
\hline $\begin{array}{l}\text { SŁOWIK et } \\
\text { al., } 2017\end{array}$ & PubMed & Polônia & 1.097 & $\begin{array}{l}\text { Analisar a qualidade de } \\
\text { vida em mulheres com } \\
\text { câncer de mama, com } \\
\text { particular ênfase nos } \\
\text { indicadores de satisfação } \\
\text { sexual, perspectivas } \\
\text { futuras e imagem } \\
\text { corporal, dependendo do } \\
\text { método de cirurgia. }\end{array}$ & $\begin{array}{l}\text { Não houve diferença significativa na qualidade de } \\
\text { vida das mulheres após realização da Cirurgia } \\
\text { Conservadora da Mama (BCT) com linfadenectomia } \\
\text { axilar. As principais mudanças relatadas referem-se } \\
\text { apenas a problemas no funcionamento sexual, mas } \\
\text { que não afetou de forma expressiva a qualidade de } \\
\text { vida geral. }\end{array}$ \\
\hline $\begin{array}{l}\text { ARCHANG } \\
\text { ELO et al., } \\
2019\end{array}$ & SciELO & Brasil & 0.0222 & $\begin{array}{l}\text { Avaliar o impacto da } \\
\text { reconstrução mamária } \\
\text { após mastectomia na } \\
\text { função sexual, imagem } \\
\text { corporal e depressão. }\end{array}$ & $\begin{array}{l}\text { Mulheres no grupo que fizeram a mastectomia } \\
\text { relataram piora significativa na função sexual, } \\
\text { surgimento de sintomas depressivos e imagem } \\
\text { corporal, sobretudo, da parte inferior do corpo. }\end{array}$ \\
\hline $\begin{array}{l}\text { TARKOWS } \\
\text { KA et al., } \\
2020\end{array}$ & PubMed & Polônia & $\begin{array}{l}\text { Não } \\
\text { disponível }\end{array}$ & $\begin{array}{l}\text { Avaliar o funcionamento } \\
\text { sexual de pacientes } \\
\text { submetidas à } \\
\text { mastectomia, cinco anos } \\
\text { após a cirurgia, em } \\
\text { comparação com um } \\
\text { grupo controle. }\end{array}$ & $\begin{array}{l}\text { Após aplicação de questionário para avaliar o } \\
\text { funcionamento sexual das mulheres, foi observado } \\
\text { que os priores resultados foram daquelas que } \\
\text { realizaram a mastectomia, citando-se a piora } \\
\text { significativa na satisfação e funcionamento sexual. } \\
\text { Isso reflete na redução do desejo, excitação, } \\
\text { lubrificação e na capacidade de atingir o orgasmo. }\end{array}$ \\
\hline $\begin{array}{l}\text { BERHILI et } \\
\text { al., } 2018\end{array}$ & PubMed & Marrocos & 2.647 & $\begin{array}{l}\text { Detectar os fatores } \\
\text { associados ao sofrimento } \\
\text { psíquico (DP) em } \\
\text { pacientes jovens } \\
\text { marroquinas com câncer } \\
\text { de mama, com ênfase } \\
\text { especial no tipo de } \\
\text { procedimento cirúrgico. }\end{array}$ & $\begin{array}{l}\text { Estudo realizado com } 122 \text { mulheres observou que a } \\
\text { partir da Escala Hospitalar de Ansiedade e Depressão } \\
\text { (HAD), que avalia o estado psicológico, } 41 \text { pacientes } \\
\text { apresentaram escore } \geq 15 \text { e } 10 \text { apresentaram escore } \\
\geq 19 \text {. Esses valores demonstram um estado } \\
\text { psicológico prejudicados, sobretudo após } \\
\text { mastectomia. }\end{array}$ \\
\hline
\end{tabular}




\begin{tabular}{|c|c|c|c|c|c|}
\hline $\begin{array}{l}\text { SEGURA et } \\
\text { al., } 2017\end{array}$ & $\begin{array}{l}\text { ScienceDi } \\
\text { rect }\end{array}$ & Espanha & 0.646 & $\begin{array}{l}\text { Desenvolver um sistema } \\
\text { de categorias de } \\
\text { conteúdo para analisar } \\
\text { construções corporais em } \\
\text { pacientes com câncer de } \\
\text { mama a partir de sua } \\
\text { própria experiência. }\end{array}$ & $\begin{array}{l}\text { O estudo trabalhou seis categorias, sendo elas: } \\
\text { Aparência Objetiva, Estética, Função, Força, Energia } \\
\text { e Emoções. Devem-se avaliar essas categorias em } \\
\text { mulheres mastectomizadas para enfocar a } \\
\text { psicoterapia nas que apresentarem desfechos mais } \\
\text { negativos, possibilitando uma imagem corporal } \\
\text { integrada após cirurgia. }\end{array}$ \\
\hline $\begin{array}{l}\text { PRATES et } \\
\text { al., } 2017\end{array}$ & SciELO & Brasil & 0.0588 & $\begin{array}{l}\text { Investigar a autoestima } \\
\text { de mulheres com e sem } \\
\text { câncer de mama em } \\
\text { relação à imagem } \\
\text { corporal. }\end{array}$ & $\begin{array}{l}\text { As mulheres sem CA de mama estavam mais } \\
\text { satisfeitas com a imagem corporal. Enquanto as que } \\
\text { fazem quimioterapia neoadjuvante mostraram-se } \\
\text { mais insatisfeitas com a aparência do que aquelas } \\
\text { com CA e sem tratamento. Logo, teve-se a } \\
\text { mastectomia como a responsável por mais } \\
\text { insatisfação com a aparência em comparação com as } \\
\text { mulheres que fizeram terapia conservadora. }\end{array}$ \\
\hline $\begin{array}{l}\text { CIEŚLAK; } \\
\text { GOLUSINS, } \\
2018\end{array}$ & $\begin{array}{l}\text { ScienceDi } \\
\text { rect }\end{array}$ & Polônia & 1.07 & $\begin{array}{l}\text { Lidar com a perda de } \\
\text { capacidade depende de } \\
\text { autoestima e controle } \\
\text { emocional? }\end{array}$ & $\begin{array}{l}\text { Após aplicação do Questionário sobre como lidar } \\
\text { com a perda de capacidade QCAL, o grupo } 1 \\
\text { mostrou-se bastante satisfeito, apresentando uma } \\
\text { autoestima alta. Quanto mais depressão o indivíduo } \\
\text { experimenta, menor é o seu nível de autoestima. No } \\
\text { grupo 2, por sua vez, observou-se que quanto maior o } \\
\text { nível de depressão, menor é o nível de raiva. }\end{array}$ \\
\hline
\end{tabular}

Fonte: Autores (2021).

A descoberta do CA de mama adjacente ao procedimento de mastectomia repercute significativamente na autoestima e identidade sexual da mulher. Este fato é consequência da representatividade que a mama possui sob a figura feminina, o qual pode levar a um conjunto de sentimentos negativos à sua feminilidade, e nas percepções acerca da atratividade, sexualidade, maternidade e de sua imagem corporal (Rocha et al., 2019; Tarkowska et al., 2020).

Diante disso, Archangelo et al. (2019), enfatizam que os procedimentos diagnósticos e intervenções cirúrgicas menos agressivas às mulheres, ampliam consideravelmente a qualidade de vida, assim como, seu tempo de sobrevida durante o enfrentamento do CA de mama. Outrossim, é imprescindível analisar os fatores biopsicossociais dos indivíduos submetidos a mastectomia, tendo em vista que após avaliação desses determinantes é possível identificar os fatores de risco capazes de interferir no tratamento e na implantação de medidas realizadas pela equipe de saúde.

Somado a isso, a extração de uma ou ambas as mamas resultam em transformações negativas à imagem corporal da mulher, repercutindo nas questões físicas e psíquicas, sobretudo, por ser um órgão sexual e de representatividade. Ademais, os danos à qualidade de vida, satisfação sexual e recreativa, assim como à autoimagem em razão da queda de cabelo e ganho de peso, são as queixas mais comuns entre as mulheres após cirurgia (Oliveira, 2019).

Em concordância, Jablonski et al. (2018), demonstraram que as mulheres examinadas, independentemente do tipo de cirurgia, revelaram valores menores no âmbito de vivência da intimidade comparado às mulheres do grupo de controle. Por conseguinte, as mulheres submetidas ao tratamento de câncer de mama mencionaram objeções no desenvolvimento afetivo com o parceiro e apresentaram nível acentuado de medo em novos encontros mediante insatisfação com seu corpo, com baixa autoestima na área de competência interpessoal.

Em contrapartida, Slowik et al. (2017), afirmam em seu estudo que não houve correlação significativa entre o tipo de cirurgia e a qualidade de vida média geral das mulheres, bem como, no funcionamento e satisfação sexual. Contudo, as mulheres após a cirurgia na mama direita apresentaram problemas no funcionamento sexual significativamente mais baixos do que as mulheres que realizaram mastectomia esquerda, podendo ser justificado devido às alterações hormonais resultantes da 
mastectomia. Assim, a retirada de qualquer uma das mamas pode resultar em impactos na autoestima, seja em mudanças comportamentais, emoções ou até mesmo na vida íntima.

A partir de um estudo comparativo entre a mastectomia e a terapia conservadora da mama observou-se que $38 \%$ das pacientes mastectomizadas relataram insatisfação sob a imagem corporal, contrapondo-se a satisfação apresentada pelas mulheres que realizaram terapia conservadora (Prates et al., 2017). Adicionalmente, evidenciou-se o surgimento de sentimentos de inferioridade e pior autopercepção do corpo, o que acarreta em sintomas de depressão, tristeza, vergonha, isolamento e visão pessimista do futuro, refletindo em alterações no cotidiano (Boing et al., 2019).

Nesse cenário, o estudo de Oliveira et al. (2019), elencam que após comprovação do CA manifestam-se efeitos variáveis consoante a história de cada indivíduo e a etapa terapêutica em que se encontram. Ao longo do tratamento, as emoções diversificam, podendo ser, a princípio, de perda, de aflição ou de aceitação. Desta forma, as acometidas procuram formas menos dolorosas de enfrentamento da doença partindo primeiramente da aceitação do CA, como uma ferramenta de defesa para adaptação da sua condição atual, e posteriormente, nas fases finais com a ideia de cura, no qual desenvolvem o sentimento de vitória e satisfação.

Em vista disso, destaca-se a importância da reconstrução mamária para refletir na redução do trauma causado pela retirada das mamas, assim como já verificado por alguns estudos, que mulheres com mamas reconstruídas após a realização da mastectomia, apresentaram-se mais satisfeitas com a sua aparência, acarretando em uma melhor qualidade de vida pósoperatória (Aygin \& Cengiz, 2018; Segura et al., 2017).

No que diz respeito à rede de apoio, a família constitui o papel primordial para o enfrentamento do diagnóstico do CA, uma vez que este suporte pode refletir positivamente na diminuição dos sintomas negativos desencadeados nas diversas situações em que as mulheres perpassam durante sua luta contra o câncer. Destaca-se ainda, o protagonismo desempenhado pelos companheiros, amigos, comunidade, bem como da equipe de saúde envolvida no processo do cuidado integral e holístico (Berhili et al., 2018; Rocha et al., 2019).

No estudo de Boing et al. (2019), o nível de escolaridade refletiu em mais sintomas depressivos, desta forma, ressaltase a importância de um sistema de educação formal, com vista a esclarecer sobre o diagnóstico e tratamento do CA de mama e principalmente, quanto a particular importância da adoção de condutas que permitam a redução de danos à sua autoestima e qualidade de vida, sugerindo-se o apoio psicológico para melhor enfrentamento. Além disso, aponta-se a relevância da partilha de experiências e atividades em grupo, como estratégias pertinentes para a obtenção de melhores resultados acerca da autoestima de mulheres mastectomizadas (Maharjan et al., 2018; Sánchez et al., 2021).

\section{Considerações Finais}

A análise realizada na presente revisão mostrou que as mamas são órgãos tidos como um símbolo da sexualidade à figura feminina, e após diagnóstico do CA de mama com necessidade da mastectomia, tornam-se vulneráveis aos diversos sentimentos negativos que surgem nesse momento. Dentre os principais sentimentos discutidos nos estudos que perpassam o diagnóstico e tratamento, destacam-se a tristeza, impotência e desconfortos físicos e emocionais pela retirada da mama.

Com vista disso, as mulheres mastectomizadas precisam ser encorajadas pela família, parceiros, equipe multiprofissional e comunidade, de modo a alterar esse estigma de mulheres mutiladas para empoderadas da sua sexualidade e do seu corpo. Portanto, ressalta-se a importância da reconstrução mamária após cirurgia, o qual é ofertado gratuitamente pelo Sistema Único de Saúde (SUS), com o intuito da autoaceitação do seu corpo, sentindo-se completas mesmo com a retirada da mama.

Em síntese, sugere-se que pesquisas futuras sejam realizadas com ênfase nas influências das variáveis socioeconômicas e ético-culturais na autoestima de mastectomizadas de modo a favorecer a adoção de políticas públicas de 
saúde voltadas a esse público em todo território nacional, tendo em vista sua influência na incidência dos cânceres e nas complicações causadas pelos mesmos.

\section{Referências}

Araujo, V. D. S. C., Oliveira Pereira, R. M., de Souza, L. O., Almeida, M. G., de Almeida, L. D. S., Silva Reis, M. H., \& Gomes, A. P. (2020). A perspectiva da autoimagem e sexualidade de mulheres mastectomizadas: revisão integrativa da literatura. Revista Eletrônica Acervo Saúde, (52), e3618-e3618.

Archangelo, S. D. C. V., Sabino Neto, M., Veiga, D. F., Garcia, E. B., \& Ferreira, L. M. (2019). Sexuality, depression and body image after breast reconstruction. Clinics, 74 .

Aygin, D., \& Cengiz, H. (2018). Life quality of patients who underwent breast reconstruction after prophylactic mastectomy: systematic review. Breast Cancer, 25(5), 497-505.

Berhili, S., Ouabdelmoumen, A., Sbai, A., Kebdani, T., Benjaafar, N., \& Mezouar, L. (2019). Radical mastectomy increases psychological distress in young breast cancer patients: Results of a cross-sectional study. Clinical breast cancer, 19(1), e160-165.

Boing, L., Pereira, G. S., Araújo, C. D. C. R. D., Sperandio, F. F., Loch, M. D. S. G., Bergmann, A., \& Guimarães, A. C. D. A. (2019). Factors associated with depression symptoms in women after breast cancer. Revista de saúde pública, 53, 30.

Cieślak, K., \& Golusiński, W. (2018). Coping with loss of ability vs. emotional control and self-esteem in women after mastectomy. Reports of Practical Oncology \& Radiotherapy, 23(3), 168-174.

Godoy, M. K., Soares, M., Guth, A. K., \& Rezer, J. F. P. (2016). Mastectomia e estética corporal: Uma revisão. XXIV Seminário de Iniciação Científica, 1-4.

Gomes, P., Lima, F. L. T., \& Santos, A. T. C. (2021). Significados da Dor Crônica na Sobrevivência ao Câncer de Mama. Revista Brasileira de Cancerologia, $67(1)$.

Instituto Nacional de Câncer. (2020). Estatísticas de câncer. https://www.inca.gov.br/numeros-de-cancer.

Instituto Nacional de Câncer. (2020). Tipos de câncer: câncer do colo do útero. https://www.inca.gov.br/tipos-de-cancer/cancer-do-colo-do-utero.

Instituto Nacional de Câncer. (2019). O que é câncer? O que causa o câncer? Como surge o câncer? http://www.inca.gov.br/o-que-e-cancer.

Jabłoński, M. J., Streb, J., Mirucka, B., Słowik, A. J., \& Jach, R. (2018). The relationship between surgical treatment (mastectomy vs. breast conserving treatment) and body acceptance, manifesting femininity and experiencing an intimate relation with a partner in breast cancer patients. Psychiatr. Pol, 52(5), $859-872$.

Junqueira, L. C. U., \& dos Santos, M. A. (2020). Atravessando a tormenta: imagem corporal e sexualidade da mulher após o câncer de mama. Revista Família, Ciclos de Vida e Saúde no Contexto Social, 8, 562-574.

Kowalczyk, R., Nowosielski, K., Cedrych, I., Krzystanek, M., Glogowska, I., Streb, J., \& Lew-Starowicz, Z. (2019). Factors affecting sexual function and body image of early-stage breast cancer survivors in Poland: a short-term observation. Clinical Breast Cancer, 19(1), e30-e39.

Maharjan, M., Thapa, N., Adhikari, R. D., Petrini, M. A., \& Amatya, K. S. (2018). Quality of Life of Nepalese Women Post Mastectomy. Asian Pacific journal of cancer prevention: APJCP, 19(4), 1005.

Menon, A. S., \& O'Mahony, M. (2019). Women's body image following mastectomy: Snap shots of their daily lives. Applied Nursing Research, 47, 4-9.

Morales-Sánchez, L., Luque-Ribelles, V., Gil-Olarte, P., Ruiz-González, P., \& Guil, R. (2021). Melhorando a autoestima e a imagem corporal de mulheres com câncer de mama por meio de intervenções: uma revisão sistemática. Jornal internacional de pesquisa ambiental e saúde pública, 18 (4), 1640.

Nascimento, K. T. S., Fonsêca, L. D. C. T., Costa Andrade, S. S., Leite, K. N. S., Costa, T. F., \& Santos Oliveira, S. H. (2015). Sentimentos e fontes de apoio emocional de mulheres em pré-operatório de mastectomia em um hospital-escola [Feelings and sources of emotional support for women in pre-operative mastectomy in a teaching hospital]. Revista Enfermagem UERJ, 23(1), 108-114.

Noal, S. J., \& Bergamaschi, D. A. (2017). Sentimentos Experimentados Por Mulheres Que Realizaram Mastectomia. Anuário Pesquisa e Extensão Unoesc São Miguel do Oeste, 2, e13006-e13006.

Oliveira, T. R., Corrêa, C. S. L., Weiss, V. F., Baquião, A. P. D. S. S., de Carvalho, L. L., Santos Grincenkov, F. R., \& Carvalho, S. M. (2019). Câncer de mama e imagem corporal: impacto dos tratamentos no olhar de mulheres mastectomizadas. Saúde e Pesquisa, 12(3), $451-462$.

Pereira, A. S., Shitsuka, D. M., Parreira, F. J., \& Shitsuka, R. (2018). Metodologia da pesquisa científica.

Prates, A. C. L., Freitas-Junior, R., Prates, M. F. O., D'Alessandro, A. A. B., \& Veloso, M. D. F. (2014). Indicadores de insatisfação relacionados à imagem corporal em pacientes submetidas à mastectomia. Revista Brasileira de Mastologia, 24 (1), 23-28.

Prates, A. C. L, Freitas-Junior, R., Prates, M. F. O, Veloso, M. D. F, \& Barros, NDM (2017). Influência da imagem corporal em mulheres em tratamento para câncer de mama. Revista Brasileira de Ginecologia e Obstetrícia, 39 (4), 175-183.

Ribeiro, F. F., \& Marteleto, R. M. (2018). A Configuração das Redes Sociais de Suporte a Pessoas com Câncer: um Olhar sob o Prisma da Informação e Comunicação em Saúde. Revista Brasileira de Cancerologia, 64(1), 77-85. 
Research, Society and Development, v. 10, n. 6, e3910716258, 2021

(CC BY 4.0) | ISSN 2525-3409 | DOI: http://dx.doi.org/10.33448/rsd-v10i7.16258

Rocha, C. B., Fontenele, G. M. C., Macêdo, M. S., de Carvalho, C. M. S., Fernandes, M. A., Veras, J. M. D. M. F., \& Soares, J. (2019). Sentimentos de mulheres submetidas à mastectomia total. Revista Cuidarte, 10(1), 4.

Santos, I. D. D. L., Alvares, R. B., Lima, N. D. M., Mattias, S. R., Cestari, M. E. W., \& Pinto, K. R. T. D. F. (2017). Câncer de mama: o apoio recebido no enfrentamento da doença. Rev. enferm. UFPE on line, 3222-3227.

Segura-Valverde, M., Saúl, L. A., \& Baca-García, E. (2017). Content categories system for body constructs applied to patients with mastectomy. The European Journal of Psychiatry, 31(2), 66-72.

Słowik, A. J., Jabłoński, M. J., Michałowska-Kaczmarczyk, A. M., \& Jach, R. (2017). Evaluation of quality of life in women with breast cancer, with particular emphasis on sexual satisfaction, future perspectives and body image, depending on the method of surgery. Psychiatr Pol, 51(5), 871-88.

Souza, M. T. D., Silva, M. D. D., \& Carvalho, R. D. (2010). Revisão integrativa: o que é e como fazer. Einstein 8(1), 102-106.

Tarkowska, M., Głowacka-Mrotek, I., Nowikiewicz, T., Monastyrska-Waszak, E., Gastecka, A., Goch, A., \& Zegarski, W. (2020). Sexual functioning and self-esteem in women after mastectomy-a single-centre, non-randomised, cross-sectional study. Contemporary Oncology, 24(2), 106. 